\title{
Kinematic Quantification of Gait Asymmetry Based on Characteristics of Angle-Angle Diagrams
}

\author{
Patrik Kutilek ${ }^{1}$, Slavka Viteckova ${ }^{1}$, \\ Zdenek Svoboda $^{2}$, Vladimir Socha ${ }^{1}$, Pavel Smrcka ${ }^{1}$
}

${ }^{1}$ Faculty of Biomedical Engineering, Czech Technical University in Prague nam. Sitna 3105, 27201 Kladno, Czech Republic, e-mail: kutilek@fbmi.cvut.cz, vitecsla@fbmi.cvut.cz, sochavla@fbmi.cvut.cz,smrcka@fbmi.cvut.cz

${ }^{2}$ Faculty of Physical Culture, Palacky University of Olomouc

Tr. Miru 115, 77111 Olomouc, Czech Republic, e-mail: zdenek.svoboda@upol.cz

Abstract: Our work focuses on studying gait symmetry with the use of bilateral angle-angle diagrams. The geometric characteristics of angle-angle diagrams can be used to characterize gait, as well as, identify and quantify the associated gait asymmetry. The angle-angle diagrams were created for ten patients with leg length inequality (LLI), to quantify gait symmetry before and after an application of the heel lift. In order to quantify the symmetry of human walking, we have invented and used the characteristics of an angleangle diagram, which represent the evolution of the two joint angles, i.e. left and right knee angles or left and right hip angles. The novel methods are based on the area of the convex hull of the hip-hip diagram or knee-knee diagram, and can be used as an additional method for the determination of gait symmetry of the bipedal walking of human subjects or robots. Our method is not limited to gait studies, it can also be used to study arm swing symmetry.

Keywords: human walking; bipedal walking; convex hull; angle-angle diagram; length inequality

\section{Introduction}

Currently, several methods can be used in medical practice and in physiotherapeutic research to identify defects in bipedal walking. The most widely-used method for studying gait behavior in clinical practice is gait phase analysis by phase cycles of gait, [1] - [4]. Intensive research is now being done on studying leg movements by EMG signal measurements [5] - [7]. For the study of gait, we have used new methods based on the analysis of gait angles using angleangle diagrams (also called cyclograms). The first mention of the cyclogram [8] 
argued that a cyclic process such as walking can be better understood if studied with a cyclic plot. The creation of cyclograms is based on gait angles that are objective, reliable and well suited for statistical study [9] - [14].

We will focus on the use of the cyclogram for the evaluation of gait symmetry. Symmetry is an important indicator of a healthy gait or proper walking technique. Human gait symmetry can be influenced by various factors, such as limb asymmetry, injury and other mobility-affecting diseases. Several methods can be used for identifying defects in bipedal walking. At present, algebraic indices and statistical parameters represent two major classes of gait symmetry quantifiers [15] - [19]. Algebraic indices include a symmetry index and a ratio index. Symmetry and ratio indices compare bilateral variables such as, step period, maximum joint angle or step length. In the case of the evaluation of maximum joint angles, the basic formula is as follows:

$$
\mathrm{R}=\frac{X}{Y},
$$

where $\mathrm{R}$ is the ratio index related to the maximum angles (maximum right angle $X$ and maximum left angle $Y$ ). Notwithstanding, their successful use depends on discrete variables and are unable to reflect asymmetry, as it evolves over a complete gait cycle. Statistical methods such as paired t-test and Principal Component Analysis (PCA), and parameters such as correlation coefficients, coefficient of variation and variance ratio have also been used to measure gait asymmetry [14], [20]. Statistical parameters do not usually suffer from the limitations of the algebraic indices, however, their computation is more complex and interpretation is less transparent. Despite the broad agreement in the fundamentals of what constitutes symmetry, there is no appropriate measure of gait symmetry (reflecting asymmetry as it evolves over a complete gait cycle) [14], [15]. In paper [14], a technique was introduced based on the geometric properties of symmetric angle-angle diagrams. This technique is rooted in geometry and the symmetry measures are intuitively understandable. Depending on the cyclicity of the gait, cyclograms are closed trajectories generated by simultaneously plotting two (or more) joint quantities. In order to quantify the symmetry of bipedal walking, a cyclogram was obtained in the study [14], representing the same joint and two sides of the body, i.e. the evolution of the two (left and right) joint angles. The presented approach is based on the symmetry of joint angle evolution and comparison of the evolution of a single joint with that of its contra-lateral counterpart, [14], [21]. The papers also mentioned basic characteristics of the bilateral cyclogram, which can be used to evaluate symmetry. The area within the cyclogram, its orientation and its minimum moment are geometric parameters used to evaluate the symmetry, [14], [22], [23]. For the perfectly (i.e. absolutely) symmetrical gait, there is a zero area within the curve, its orientation is 45 degrees and the magnitude of its minimum geometric moment is zero [14]. However, angle-angle diagrams have never been used for 
studying patients with Leg Length Inequality (LLI), hence the new shape characteristics of angle-angle diagrams can be very useful. Therefore, the main object of our study was to test and verify the application of the method based on the area of convex hull of angle-angle diagram to analyze the gait symmetry of bipedal walking of patients with LLI.

\section{Methods and Materials}

\subsection{Participants and Test Procedure}

The set of data to create and study angle-angle diagrams was measured on a sample of 10 volunteers/adult patients (age of 50 (SD 14.8) years) with leg length inequality (leg-length inequality of $0.85(\mathrm{SD} 0.32) \mathrm{mm}$ ) recruited from patients of the Rehabilitation Center Kladruby (Kladruby u Vlasimi, the Czech Republic). The subjects were asked to walk properly on a treadmill. A human walking speed of $2.3 \mathrm{~km} / \mathrm{h}$ was selected for studying the proposed method. A constant speed was chosen because it directly affects the range of joint angles, [24], [25]. If we want to evaluate and compare shape characteristics of the angle-angle diagram, it is appropriate to measure the joint angles at a constant walking speed. We assume that improvement or deterioration of gait symmetry is not primarily influenced by walking speed.

First, patients were asked to walk without shoes and any support devices, while their joint angles (left/right knee angles and left/right hip angles) were measured. After that, the physician recommended appropriate shoes with a heel lift under the short leg side to stabilize and level the pelvis. Patients were asked to walk immediately after the application of the heel lift, and the joint angles were measured again.

\subsection{Motion Capture Equipment}

To create and study angle-angle diagrams, we used a model of the human body created in MatLab Simulink and SimMechanic software, [26]. The movement of the model of a body is controlled by data measured by the motion capture system (MoCap), which identifies the position of body segments in the Cartesian coordinate system. As a MoCap system, we used a very accurate Lukotronic AS 200 system (Lutz Mechatronic Technology declares, according to the EU Directive 93/42/EEC, that the system can be used in hospitals) with IR active markers placed on the following anatomical points on the person being measured: fifth metatarsophalangeal joint, malleolus lateralis, epicondylus lateralis, 
trochanter major and spina iliaca anterior superior, Fig. 1, [16], [27]. The markers fastened with small adhesive double-sided tape were placed in accordance with the recommendation of the manufacturer of the MoCap system. Using this method, we were able to record and study the movement in 3D space, though we primarily studied the movement in a $2 \mathrm{D}$ sagittal plane. Maximum joint angles are small in the frontal plane and thus, one of the basic tools to study the gait is the analysis of the joint angles in the sagittal plane, [28], [29]. The MoCap system was calibrated accurately before the experiments, and the origin of the world coordinate system was set up so that the first axis is along the track of the treadmill and the other two axes are perpendicular to the track of the treadmill.

After obtaining the measured data, we identified joint angles and created the cyclograms in MatLab (The MathWorks Inc.) software. If we have information on the movement of anatomical points/markers in space, and the points characterizing the parts of human body, then we can use these to define the vectors of the positions and orientations of body segments. The difference in coordinates of two points in space defines a vector. The angles between each two segments are calculated by assuming the segments to be idealized rigid bodies. The angles are given by formula described in detail by Kutilek et al. [22].
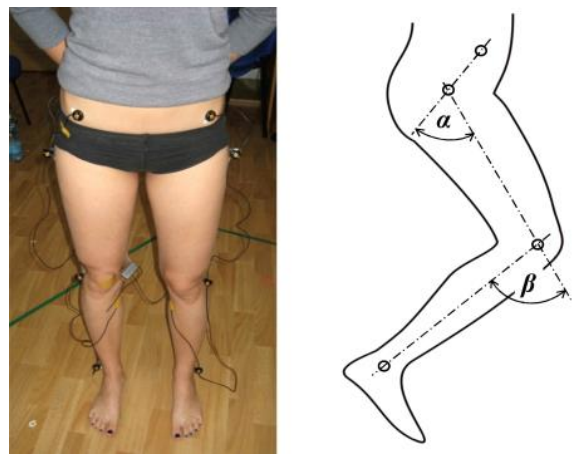

Figure 1

Placement of IR markers and angles measured during the examination, [22]

\subsection{Gait Evaluation Methods}

After the measurement and data processing, we used the method based on synchronization of two angle plots [14] to obtain bilateral knee-knee and hip-hip cyclograms. A prominent gait event such as the heel-touchdown was used to synchronize the two plots representing angles at the left and right joint. For a symmetric gait, properly synchronized twin trajectories from corresponding joints should be identical and a bilateral cyclogram should lie on a symmetry line, [14]. The symmetry line is a straight line passing through the origin inclined at an angle 45 degrees, see Fig. 2. We can mathematically measure cyclogram deviation from 
the symmetry line to obtain a quantification of gait symmetry. We can also use the area within the cyclogram and its orientation to evaluate the symmetry, [14].

A bilateral cyclogram is not continuous curve, but it's a polyline contour (i.e. irregular polygon), see Fig. 2. The smoothness of the contour is a function of the amount of noise in the measured data. We found that in some cases, the evaluation of the cyclogram using the area within the cyclogram and its orientation is incorrect. The cyclograms may consist of self-intersecting loops or they have a complex shape. Although the cyclogram may be complex and asymmetrical, its surface may also be very small. For example, a crescent shape can lead to a small area, although it is an asymmetrical gait, see Fig. 2.

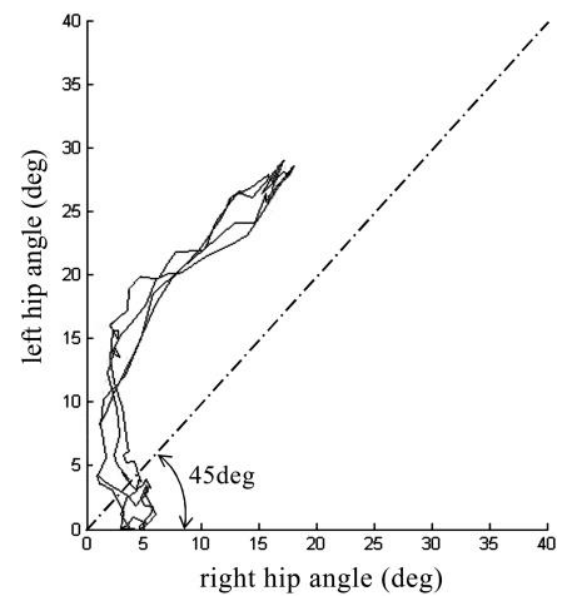

Figure 2

Example of a right hip vs. left hip bilateral cyclogram for measured patient with LLI, deliberately unfiltered, with symmetry line

For the above mentioned reason, we use the convex hull to calculate the area which represents the shape of the cyclogram. The convex hull of a cyclogram is more appropriate to describe the area which represents the gait asymmetry. A convex hull completely encloses a set of points with the fewest number of perimeter nodes. In our case, the set of points consists of all points on the cyclogram. The area of convex hull is usually larger than the area within the cyclogram if the cyclogram is asymmetric and with loops, see Fig. 3a. The second way to avoid improper evaluation of the area is based on the calculation of the area of the region enclosed by the curve of the cyclogram, the $45^{\circ}$ straight line (symmetry line) and the lines perpendicular to the symmetry line, see Fig. 3b. The region is represented by the area above and below the symmetry line and is bounded by the outer curves of the cyclogram and lines perpendicular to the symmetry line. The positions of perpendicular lines are defined by the points of intersection on the symmetry line. The points are identified by projection of the points of the bounding curve of the cyclogram on the symmetry line, and are the 
nearest and the furthest points of the projection of the cyclogram above/below the symmetry line from the origin of the symmetry line, Fig. $3 \mathrm{~b}$. We can also use the convex hull and/or the region enclosed by the curve of the cyclogram and symmetry line to calculate the second moment of area to describe the property of a two-dimensional plane shape of the cyclogram. Therefore, we can determine the second moment of area about the symmetry line.

The orientation of the planar geometric entity, i.e. convex hull and/or the region enclosed by the curve of the cyclogram and symmetry line, can be defined as the angle (bounded by $+/-90^{\circ}$ ) between the symmetry line and the principal axis of the planar geometric entity, [14]. We can also use linear regression to determine the orientation of the planar geometric entity. Simple linear regression fits a straight line (axis) through the set of the points on the cyclogram, or only through the set of the points of bounding curve. Hence, the orientation can be defined as the angle between the regression principal axis and the symmetry line, [27].
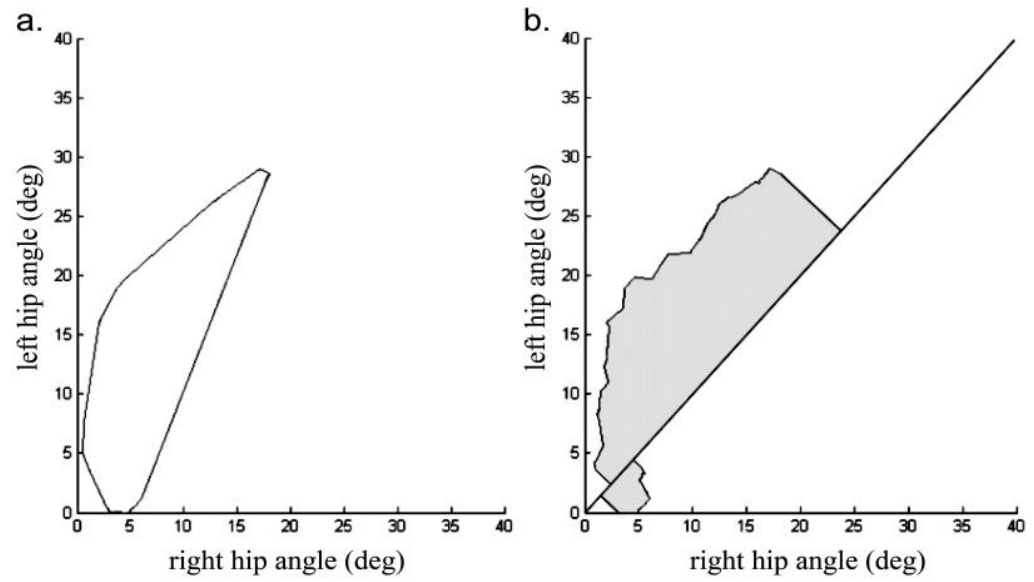

Figure 3

a) Example of the convex hull of the bilateral cyclogram (see Fig. 1); b) Example of the area of the region enclosed by the curve of the cyclogram (see Fig. 1) with symmetry line

The described symmetry quantification technique, based on convex hull and/or the region enclosed by the curve of the cyclogram, is sensitive to the precision of synchronization of the two signals. The area of the convex hull and/or the region enclosed by the curve of the cyclogram dramatically increases from zero for even a small time-shift, i.e. poor synchronization of the of two angle plots. In the next section, we describe the application of the method based on the area of convex hull. This method is easier and computationally faster than other methods/algorithms. The technique is also intuitively understandable.

We used convhull (i.e. MatLab function) to create convex hull of each bilateral cyclogram. The convhull, [30], returns the 2-D convex hull [31], of the points defined by coordinates $(x, y)$, where $x$ and $y$ are values of the left joint angle and 
right joint angle. The convhull function is based on Quick Hull method. This method computes the convex hull of a finite set of points in the plane. It uses a "divide and conquer" approach similar to that of a QuickSort sorting algorithm. We also used MatLab to calculate the area of the convex hull of each bilateral cyclogram.

The symmetry index (SI) was used as a comparative method to compare and quantify the symmetry of bipedal walking, [32]. The SI is a measure of the relationship between the discrete values obtained from the left and right sides. We used the "average" symmetry index:

$\mathrm{SI}_{\text {average }}=\frac{2 \cdot|X-Y|}{|X+Y|} \cdot 100 \%$

The symmetry index is related to the maximum angles (maximum right angle $X$ and maximum left angle $Y$ ) formed when the right-side value is plotted and the left-side value is plotted. After the measurements, the area of convex hulls of cyclograms and the symmetry indexes were calculated. We assume that the decrease of the area of the convex hull indicates the improvement in symmetry of bipedal walking and the as well as the decrease of the symmetry index indicates the improvement in symmetry of bipedal walking, [33].

\section{Results}

The calculated areas of convex hulls and symmetry indexes are summarized in Table 1 and Table 2. The tables compare the area of convex hulls and symmetry indexes before the application of the heel lift and after the application of the heel lift. Table 1 represents the area of the convex hull and symmetry index of the right knee angle and left knee angle, and Table 2 represents the area of the convex hull and symmetry index of the right hip angle and left hip angle before and after the application of heel lift.

We used the measured data (Table 1 and Table 2) to test the proposed method and illustrate the relationship between the area of convex hull of the bilateral cyclogram and the symmetry index. The following plots (Fig. 4 and Fig. 5) display the minimum, maximum, median, first quartile (Q1), third first quartile (Q3), mean and standard deviation (SD) for the area of the convex hull of kneeangle cyclograms and hip-angle cyclograms before and after the application of heel lift, and the SI of knee angles and hip angles before and after the application of heel lift.

The data measured before and after the application of the heel lift indicates a decrease of the area of the convex hull of the knee-knee cyclogram in only three cases. The data also indicates a slight increase of the mean for the area of the 
convex hulls, Tab. 3, Fig. 4. The gait symmetry index related to the maximum left and right knee angles decreased in five cases. The data indicate a slight increase of the mean for the SI, Tab. 4, Fig. 5. Improvements in the two characteristics, which describe the gait symmetry, are overlapping only in one case (i.e. 10\%), Table 1.

Table 1

Area of the convex hull and symmetry index of the right knee angle and left knee angle before and after the application of the heel lift

\begin{tabular}{|c|c|c|c|c|}
\hline \multirow{2}{*}{$\begin{array}{l}\text { patient with } \\
\text { LLI }\end{array}$} & \multicolumn{2}{|c|}{ Area of convex hull $\left[\mathrm{deg}^{2}\right]$} & \multicolumn{2}{|c|}{ Symmetry index [\%] } \\
\hline & $\begin{array}{c}\text { before } \\
\text { application }\end{array}$ & $\begin{array}{c}\text { after } \\
\text { application }\end{array}$ & $\begin{array}{c}\text { before } \\
\text { application }\end{array}$ & $\begin{array}{c}\text { after } \\
\text { application }\end{array}$ \\
\hline 1. & 884 & 733 & 13.4 & 14.3 \\
\hline 2. & 335 & 581 & 16.8 & 16.4 \\
\hline 3. & 567 & 526 & 18.6 & 28.7 \\
\hline 4. & 326 & 457 & 26.3 & 22.3 \\
\hline 5. & 841 & 610 & 57.7 & 55.3 \\
\hline 6. & 409 & 621 & 1.6 & 10.3 \\
\hline 7. & 696 & 785 & 1.4 & 3.3 \\
\hline 8. & 723 & 1098 & 29.7 & 22.9 \\
\hline 9. & 510 & 630 & 46.4 & 56.7 \\
\hline 10. & 386 & 553 & 38.6 & 24.9 \\
\hline
\end{tabular}

Table 2

Area of the convex hull and symmetry index of the right hip angle and left hip angle before and after the application of heel lift

\begin{tabular}{|c|c|c|c|c|}
\hline \multirow{2}{*}{$\begin{array}{l}\text { patient with } \\
\text { LLI }\end{array}$} & \multicolumn{2}{|c|}{ Area of convex hull $\left[\mathrm{deg}^{2}\right]$} & \multicolumn{2}{|c|}{ Symmetry index [\%] } \\
\hline & $\begin{array}{c}\text { before } \\
\text { application }\end{array}$ & $\begin{array}{c}\text { after } \\
\text { application }\end{array}$ & $\begin{array}{c}\text { before } \\
\text { application }\end{array}$ & $\begin{array}{c}\text { after } \\
\text { application }\end{array}$ \\
\hline 1. & 168 & 163 & 8.1 & 5.6 \\
\hline 2. & 123 & 135 & 5.3 & 2.1 \\
\hline 3. & 143 & 160 & 25.2 & 55.6 \\
\hline 4. & 142 & 171 & 0.5 & 1.3 \\
\hline 5. & 397 & 343 & 50.6 & 23.0 \\
\hline 6. & 235 & 348 & 16.5 & 6.7 \\
\hline 7. & 210 & 154 & 68.0 & 53.9 \\
\hline 8. & 154 & 271 & 4.5 & 12.8 \\
\hline 9. & 225 & 212 & 20.7 & 0.1 \\
\hline 10. & 223 & 201 & 13.0 & 1.4 \\
\hline
\end{tabular}

The data measured before and after the application of the heel lift indicate a decrease of the area of the convex hull of the hip-hip cyclogram in five cases. The data indicate a slight increase of the mean of the area of the convex hulls, Tab. 3, Fig. 4. The gait symmetry index related to the maximum left and right hip angles 
decreased in seven cases. The data indicate a decrease of the mean of the SI, Tab. 4, Fig. 5. Improvements in the two characteristics, which describe the gait symmetry, are overlapping in five cases (i.e. 50\%), Table 2.

Table 3

Comparison of the area of the convex hull of knee-angle cyclograms and hip-angle cyclograms before and after the application of the heel lift

\begin{tabular}{|c|c|c|c|c|}
\cline { 2 - 5 } \multicolumn{1}{c|}{} & $\begin{array}{c}\text { Area (hip angles, } \\
\text { before treatment) } \\
{\left[\mathrm{deg}^{2}\right]}\end{array}$ & $\begin{array}{c}\text { Area (hip angles, } \\
\text { after treatment) } \\
{\left[\mathrm{deg}^{2}\right]}\end{array}$ & $\begin{array}{c}\text { Area (knee } \\
\text { angles, before } \\
\text { treatment) }\left[\mathrm{deg}^{2}\right]\end{array}$ & $\begin{array}{c}\text { Area (knee } \\
\text { angles, after } \\
\text { treatment) }\left[\mathrm{deg}^{2}\right]\end{array}$ \\
\hline Mean & 202.00 & 215.80 & 567.70 & 659.40 \\
\hline SD & 79.53 & 78.31 & 208.01 & 180.91 \\
\hline Median & 189.00 & 186.00 & 538.50 & 615.50 \\
\hline Q1 & 145.75 & 160.75 & 391.75 & 560.00 \\
\hline Q3 & 224.50 & 256.25 & 716.25 & 707.25 \\
\hline
\end{tabular}

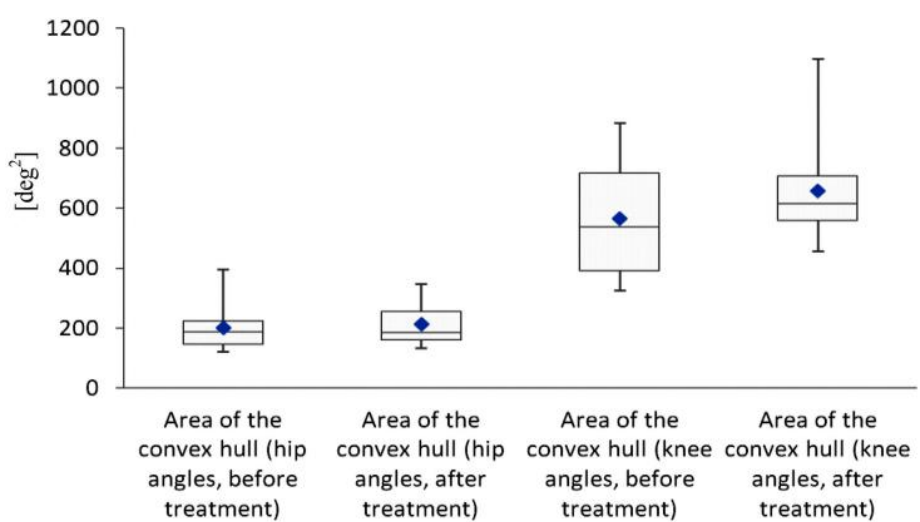

Figure 4

Comparison of the area of the convex hull of knee-angle cyclograms and hip-angle cyclograms before and after the application of the heel lift

The results of the comparison indicate that the methods show slightly different results. The evaluation method based on the area of convex hull shows improvement of the gait symmetry in fewer cases than the method based on the gait symmetry index. The reason is that the symmetry index depends on discrete variables and is thus unable to reflect the asymmetry, as it evolves over a complete gait cycle. The area of the convex hull depends on the complete gait cycle, i.e. relations and evolutions of joint angles over time, Fig. 6, which shows the bilateral cyclogram with large area of the convex hull and low SI. The SI is low because the maximum measured left and right hip angles are almost the same. However, we can see different sizes of the joint angles during specific phases of the gait cycle and the irregular curve of the bilateral cyclogram. 
Table 4

Comparison of the SI of knee angles and hip angles before and after the application of the heel lift

\begin{tabular}{|c|c|c|c|c|}
\cline { 2 - 5 } \multicolumn{1}{c|}{} & $\begin{array}{c}\text { SI (hip angles, } \\
\text { before treatment) } \\
{[\%]}\end{array}$ & $\begin{array}{c}\text { SI (hip angles, } \\
\text { after treatment) } \\
{[\%]}\end{array}$ & $\begin{array}{c}\text { SI (knee angles, } \\
\text { before treatment) } \\
{[\%]}\end{array}$ & $\begin{array}{c}\text { SI (knee angles, } \\
\text { after treatment) } \\
{[\%]}\end{array}$ \\
\hline Mean & 21.24 & 16.25 & 25.05 & 25.51 \\
\hline SD & 21.84 & 21.43 & 18.51 & 17.71 \\
\hline Median & 14.75 & 6.15 & 22.45 & 22.60 \\
\hline Q1 & 6.00 & 1.58 & 14.25 & 14.83 \\
\hline Q3 & 24.08 & 20.45 & 36.38 & 27.75 \\
\hline
\end{tabular}

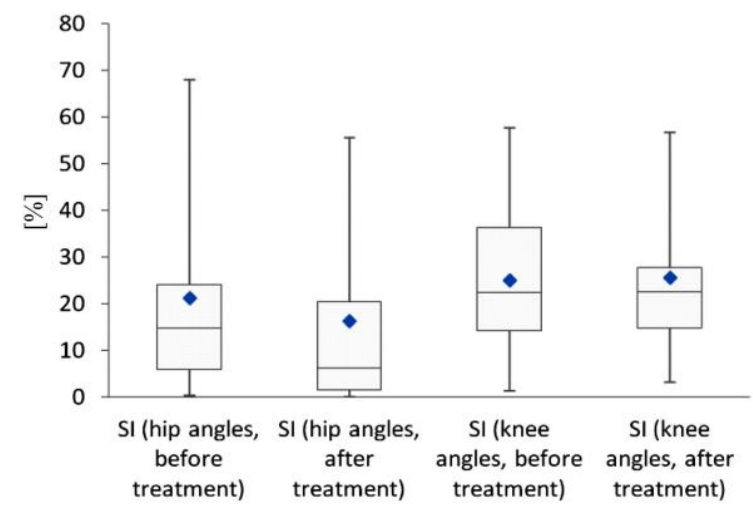

Figure 5

Comparison of the SI of knee angles and hip angles before and after the application of the heel lift

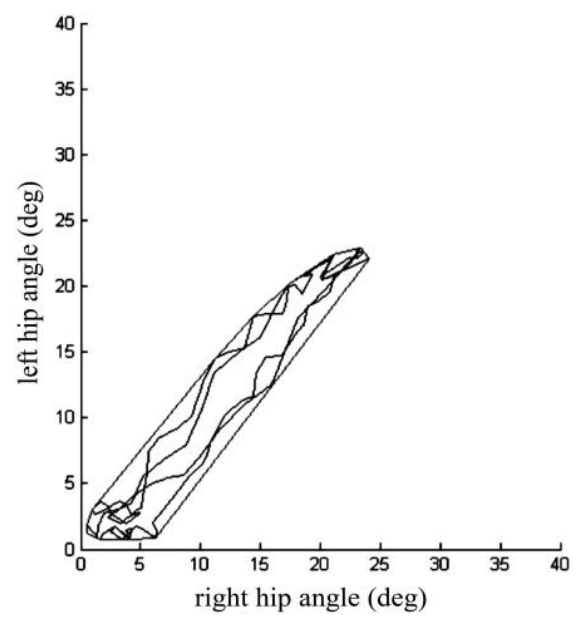

Figure 6

Example of right hip vs. left hip bilateral cyclogram (unfiltered data) with large area of the convex hull and supposed low SI 
The measured data indicate that the improvement of the symmetry, i.e. the decrease of the area of the convex hull and symmetry index, is more pronounced in the case of hip angle measurements. In the case of knee angle measurements, we observed a small improvement of the symmetry. The data indicates a slight increase of the mean for the area of the convex hulls. Generally, the decrease of the area of the convex hull and symmetry index is up to $50 \%$ of the measured subjects.

\section{Discussion}

We tested and verified a new method, which is derived from the geometric properties of angle-angle diagrams. The described symmetry quantification technique based on the area of the convex hull of the bilateral cyclogram is sensitive to the precision of synchronization of the two signals and their quality, [32]. After obtaining the measured data, we created bilateral cyclograms using MatLab software and the MatLab functions were used to calculate the area of the convex hull for each bilateral cyclogram. As it can be seen from the examples above, it is not necessary to normalize the data because the standard ranges of angles are the same for the two sides of the body if the gait is symmetric.

We found that a study of symmetry based on an algebraic parameter (i.e. symmetry index) is not suitable for the study of complex movements, but the study of symmetry based on the bilateral cyclograms is influenced by many factors that affect accuracy and relevance, [32]. The area of the convex hull of the bilateral cyclograms can be used to study complex movements and describes the deviations of the measured angles from ideal angles. Using the area of the convex hull, we could also study, for example, the deviations of angles that are caused by a tremor. Note that algebraic indices (i.e. SI) cannot be used to identify gait asymmetry caused by a tremor, or similar movement disorders.

The results indicate that the new method, described in this work, has identified and quantified improvements of gait symmetry, but only in few cases. The method based on algebraic parameters, identified improvement of the gait symmetry after the application of a heel lift in more cases than the method based on the convex hull. The main reason for this result is the short application time of the heel lift. The subjects were measured immediately after the application of the heel lift and were not able to become accustomed to using the heel lifts. Also, the physician applied the heel lift and only maximum angles were used to evaluate the gait symmetry.

\section{Conclusions}

According to the method described here, bilateral cyclograms illustrate bipedal gait symmetry. This information is important for rehabilitation medicine, and also 
could be used in control algorithms for lower limb prostheses or in the creation of bipedal robots. The method based on the convex hull of bilateral cyclograms can be used as an additional method for the determination of gait symmetry. Thus, future work can be focused on the comparison of other traditional methods based on algebraic indices and statistical parameters with the new method. In future studies, we plan to measure more patients with other types of disabilities and evaluate gait symmetry for a longer period of therapy.

This work has not attempted to describe all of the potential ways of applying the convex hull of the bilateral cyclograms. We have demonstrated new methods that have subsequently been tested on patients with Leg Length Inequality (LLI). The modified characteristics of angle-angle diagram have never previously been described and angle-angle diagrams have never been used for the study of patients with LLI. There is no limit for the research in field mentioned herin. However, the proposed method is sensitive to the precision of synchronization of the two signals and the quality of the signals. For this reason, it is necessary to use highly accurate and high quality MoCap systems and mathematical software. This may be the greatest problem in the use of the proposed methods for clinical practice. However, this can be overcome by cost reductions in MoCap systems in the future, and their introduction into clinical practice.

\section{Acknowledgement}

The work presented here was carried out at the Czech Technical University in Prague, Faculty of Biomedical Engineering within the framework of research program No. SGS14/094/OHK4/1T/17 CTU Prague.

\section{References}

[1] Gage R. J., Hicks R.: Gait Analysis in Prosthetics. Clinical Prosthetics \& Orthotics, 1989; 9: 17-21

[2] Janura M., Cabell L., Svoboda Z., Kozáková J., Gregorkova A.: Kinematic Analysis of Gait in Patients with Juvenile Hallux Valgus Deformity. Journal of Biomechanical Science and Engineering, 2008; 3: 390-398

[3] Koktas N. S., Yalabik N., Yavuzer G.: Combining Neural Networks for Gait Classification. Proceedings of the $11^{\text {th }}$ Iberoamerican Congress on Pattern Recognition, Mexico, Cancun; 2006; 11: 381-388

[4] Wang L., Tan T., Ning H., Hu W.: Automatic Gait Recognition Based on Statistical Shape Analysis. IEEE Trans Image Process., 2003; 12: 11201131

[5] Sepulveda F., Wells D., Vaughan C.: A Neural Network Representation of Electromyography and Joint Dynamics in Human Gait. J Biomech, 1993; 26: $101-109$

[6] Heller B. W., Veltlink P. H., Rijkhof N. J. M., Rutten W. L. C., Andrevs B.: Reconstructing Muscle Activation during Normal Walking: a Comparison 
of Symbolic and Connectionist Machine Learning Techniques. Biol Cybern, 1993; 69: 327-335

[7] Arsenault A. B., Winter D. A., Marteniuk R. G.: Bilateralism of EMG Profiles in Human Locomotion. Am J Phys Med Rehabil, 1986; 65: 1-16

[8] Grieve D. W.: Gait Patterns and the Speed of Walking. Biomed Eng, 1968; 3: $119-122$

[9] Grieve D. W.: The Assessment of Gait. Physiotherapy, 1969; 55: 452-460

[10] Kutílek P., Viteckova S.: Prediction of Lower Extremity Movement by Cyclograms, Acta Polytechnica, 2012; 52: 51-60

[11] Hajny O., Farkasova B.: A Study of Gait and Posture with the Use of Cyclograms. Acta Polytechnica, 2010; 50: 48-51

[12] Giannini R. C., Perell K. L.: Lower Limb Coordination during Walking in Subjects with Post Stroke Hemiplegia vs. Healthy Control Subjects. Clinical Kinesiology, 2005; 59: 63-70

[13] Heck A., Holleman A.: Walk like a Mathematician: an Example of Authentic Education. Proceedings of ICTMT6 - New Technologies Publications, Greece, Athens; 2003

[14] Goswami A.: Kinematics Quantification of Gait Symmetry Based on Bilateral Cyclograms. Proceedings of the XIXth Congress of the International Society of Biomechanics (ISB), New Zealand, Dunedin; 2003

[15] Sadeghi H., Allard P., Prince F., Labelle H.: Gait Symmetry and Limb Dominance in Able-bodied Gait: A review. Gait Posture, 2000; 12: 34-45

[16] Legnani G., Zappa B., Casolo F., Adamini R., Magnani P. L.: A Model of an Electro-Goniometer and Its Calibration for Biomechanical Applications. Medical Engineering and Physics, 2000; 22: 711-722

[17] Herzog W., Nigg B. M., Read L. J., Olsson E.: Asymmetries in Ground Reaction Force Patterns in Normal Human Gait. Med Sci Sport Exer, 1989; 21: $110-4$

[18] Vogt L., Banzer W., Bayer I., Schmidtbleicher D., Kerschbaumer F.: Overground and Walkway Ambulation with Unilateral Hip Osteoarthritis: Comparison of Step Length Asymmetries and Reproducibility of Treadmill Mounted Force Plate Readings. Physiotherapy Theory and Practice, 2006; 22: $73-82$

[19] Roerdink M., Beek P. J.: Understanding Inconsistent Step-Length Asymmetries Gross Hemiplegic Stroke Patients: Impairments and Compensatory Gait. Neurorehab Neural Re, 2011; 25: 253-258

[20] Pierotti S. E., Brand R. A., Gabel R. H., Pedersen D. R., Clarke W. R.: Are Leg Electromyogram Profiles Symmetrical? J Orthop Res., 1991; 9: 720 729 
[21] Goswami A.: New Gait Parameterization Technique by Means of Cyclogram Moments: Application to Human Slope Walking. Gait and Posture, 1998; 8: 15-26

[22] Kutilek P., Farkasova B.: Prediction of Lower Extremities Movement by Angle-Angle Diagrams and Neural Networks. Acta of Bioengineering and Biomechanics, 2011; 13: 57-65

[23] Goldstein H.: Classical Mechanics (2 ${ }^{\text {nd }}$ ed.) Boston: Addison-Wesley; 1980

[24] Segers V., Lenoir M., Aerts P., De Clercq D.: Kinematics of the Transition between Walking and Running when Gradually Changing Speed. Gait Posture, 2007; 3: 349-61

[25] Segers V., Aerts P., Lenoir M., De Clercq D.: Spatiotemporal Characteristics of the Walk-to-Run and Run-to-Walk Transition when Gradually Changing Speed. Gait Posture; 2006; 2: 247-54

[26] Kutilek P., Hajny O.: Study of Human Walking by SimMechanics. Proceedings of the $18^{\text {th }}$ Technical Computing, Slovakia, Bratislava; 2010

[27] Kutilek P., Hozman J.: Prediction of Lower Extremities Movement Using Characteristics of Angle-Angle Diagrams and Artificial Intelligence. Proceedings of the $3^{\text {rd }}$ International Conference on E-Health and Bioengineering - EHB 2011, Romania, Iasi; 2011

[28] Su P., Gard S., Lipschutz R., Kuiken T.: Gait Characteristics of Persons with Bilateral Transtibial Amputations. Journal of Rehabilitation Research and Development; 2007; 4: 491-501

[29] Haudum A., Birklbauer J., Müller E.: The Effect of an Acute Bout of Rubber Tube Running Constraint on Kinematics and Muscle Activity. Journal of Sports Science \& Medicine, 2012; 3: 459-467

[30] Rovenski V.: Modeling of Curves and Surfaces with MATLAB, New York: Springer; 2010

[31] Adkin A. L., Bloem B. R., Allum J. H.: Trunk Sway Measurements during Stance and Gait Tasks in Parkinson's Disease. Gait Posture, 2005; 22: 240 249

[32] Zifchock R. A., Davis I., Higginson J., Royer T.: The Symmetry Angle: A Novel, Robust Method of Quantifying Asymmetry. Gait Posture, 2008; 27: 622-627

[33] Kutilek P., Viteckova S., Svoboda Z., Smrcka, P.: Kinematic Quantification of Gait Asymmetry in Patients with Peroneal Nerve Palsy Based on Bilateral Cyclograms. J Musculoskel Neuron, 2013; 2: 244-250 